\title{
When more is less: Nonmonotonic trends in adsorption on clusters in alloy surfaces
}

Abigale P. Monasterial, ${ }^{1,2}$ Calla A. Hinderks, ${ }^{1,3}$, Songkun Viriyavaree, ${ }^{1}$ Matthew M. Montemore ${ }^{1, *}$

${ }^{1}$ Department of Chemical and Biomolecular Engineering, Tulane University, New Orleans, LA

70118, USA

${ }^{2}$ Department of Chemical and Biomolecular Engineering, University of Connecticut, Storrs, CT 06269, USA

${ }^{3}$ Department of Physics and Engineering Physics, Tulane University, New Orleans, LA

70118, USA

\section{Corresponding Author \\ *mmontemore@tulane.edu}




\section{ABSTRACT}

Single-atom alloys can be effective catalysts and have been compared to supported single-atom catalysts. To rationally design single-atom alloys and other surfaces with localized ensembles, it is crucial to understand variations in reactivity when varying the dopant and the ensemble size. Here, we examined hydrogen adsorption on surfaces embedded with localized clusters and discovered general trends. Counterintuitively, increasing the amount of a more reactive metal sometimes makes a surface site less reactive. This behavior is due to the localized electronic states in many of these surfaces, making them similar to free-standing nanoclusters. Further, single-atom alloys have qualitatively different behavior than larger ensembles. Specifically, the adsorption energy is U-shaped when plotted against the dopant's group for single atom alloys. Additionally, adsorption energies on single atom alloys correlate more strongly with the dopant's p-band center than the d-band center.

\section{TOC GRAPHICS}

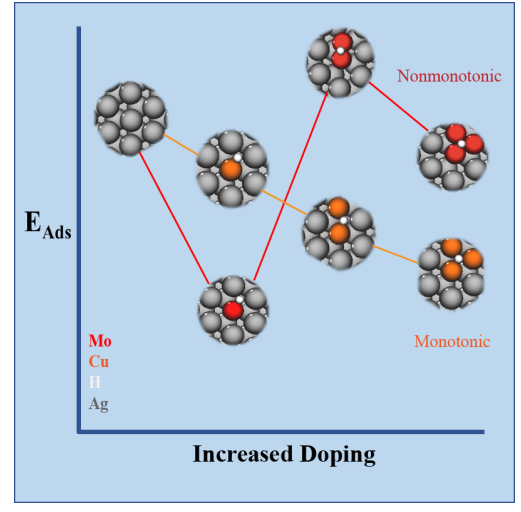

KEYWORDS Single atom alloys, adsorption, heterogeneous catalysis, nanocluster, density functional theory 
Rationalizing and designing catalysts requires a strong understanding of how active sites behave upon modification. For alloy catalysts, it is particularly crucial to understand how active site reactivity-often measured with an adsorption energy-changes upon modifying the composition of the active site. Intuitively, we expect that as the composition of a surface site is smoothly varied, the binding strength of species to the site will vary smoothly as well. Indeed, this has been shown to be the case in many systems..$^{1-5}$ For example, density functional theory (DFT) calculations show that alloying increasing amounts of Ag or Pd into Au causes the adsorption strength of $\mathrm{O}$ to smoothly decrease. Similarly, experiments of gas exposure to alloy single crystals often show smooth, monotonic changes in uptake as composition is changed. ${ }^{2,6,7}$

Small, localized ensembles of dopant atoms embedded in a host have recently gained significant attention, as they can be effective catalysts for many reactions, such as selective hydrogenation ${ }^{8-10}$ Single-atom alloys have received particular attention, including development of models and correlations for predicting or understanding adsorption energies. ${ }^{11-13}$ Single-atom alloys can have unusual properties, such as allowing spillover and breaking linear correlations between energies..$^{14-16}$ Further, they often feature localized, sharp electronic states..$^{12,17,18}$ As this is similar to the behavior of nanoclusters (small particles with only a few atoms), ${ }^{19}$ alloy surfaces with localized ensembles have been called "embedded nanoclusters". ${ }^{20}$

In this work, we show how localized clusters of atoms embedded in the surface of a host can defy intuition and link this counterintuitive behavior to the narrow d-state peaks in these systems. By studying many dopants and several hosts, we also elucidate trends in the behavior of these systems, which allows us to develop predictive correlations for single atom alloys and embedded nanoclusters. In particular, we show that single-atom alloys have qualitatively 
different behavior from dimers and trimers in how the $\mathrm{H}$ adsorption energy depends on the electronic structure.

To elucidate trends in adsorption on embedded nanoclusters, we performed DFT calculations for $\mathrm{H}$ adsorption on late transition metal fcc(111) surfaces substitutionally doped with one, two, and three metal atoms. We focused on $\mathrm{Ag}$ as the host element, but also examined $\mathrm{Au}, \mathrm{Cu}$, and $\mathrm{Pd}$ as hosts for comparison, and we included 19 other d-block metals (Co, $\mathrm{Cr}, \mathrm{Fe}$, Hf, Ir, Mn, Mo, Ni, Pt, Re, Rh, Ru, Sc, Ta, Tc, Ti, V, W, Zn) as guests. We denote $n$ dopants of a guest $\mathrm{X}$ dopant into a host $\mathrm{Y}$ as $\mathrm{X}_{\mathrm{n}} \mathrm{Y}$, such that $\mathrm{Cu}_{1} \mathrm{Ag}$ denotes a single $\mathrm{Cu}$ atom doped into a $\operatorname{Ag}(111)$ surface.

Doping a single heteroatom into a Ag surface nearly always strengthens $\mathrm{H}$ adsorption, as Ag binds $\mathrm{H}$ relatively weakly. Intuitively, we generally expect that adding more of the reactive metal to the adsorption site will further strengthen $\mathrm{H}$ adsorption, as it can now bond to more atoms of that metal. In other words, intuition suggests that the trends should be monotonic, with adsorption energies varying roughly linearly with the number of dopants added. This is indeed the case for $\mathrm{Cu}$ (Figure 1a): adding increasing amounts of $\mathrm{Cu}$ to the adsorption site stabilizes $\mathrm{H}$ approximately linearly. However, for Mo, adding a second dopant atom significantly weakens $\mathrm{H}$ adsorption compared to the single-Mo case, to such a degree that $\mathrm{Mo}_{2} \mathrm{Ag}$ adsorbs $\mathrm{H}$ even weaker than pure $\mathrm{Ag}$. This is a radical departure from intuition. Similarly, adsorption on $\mathrm{W}_{\mathrm{n}} \mathrm{Ag}$ follows a monotonically decreasing trend when up to two dopants are added, but breaks this trend when a third dopant atom is added. 

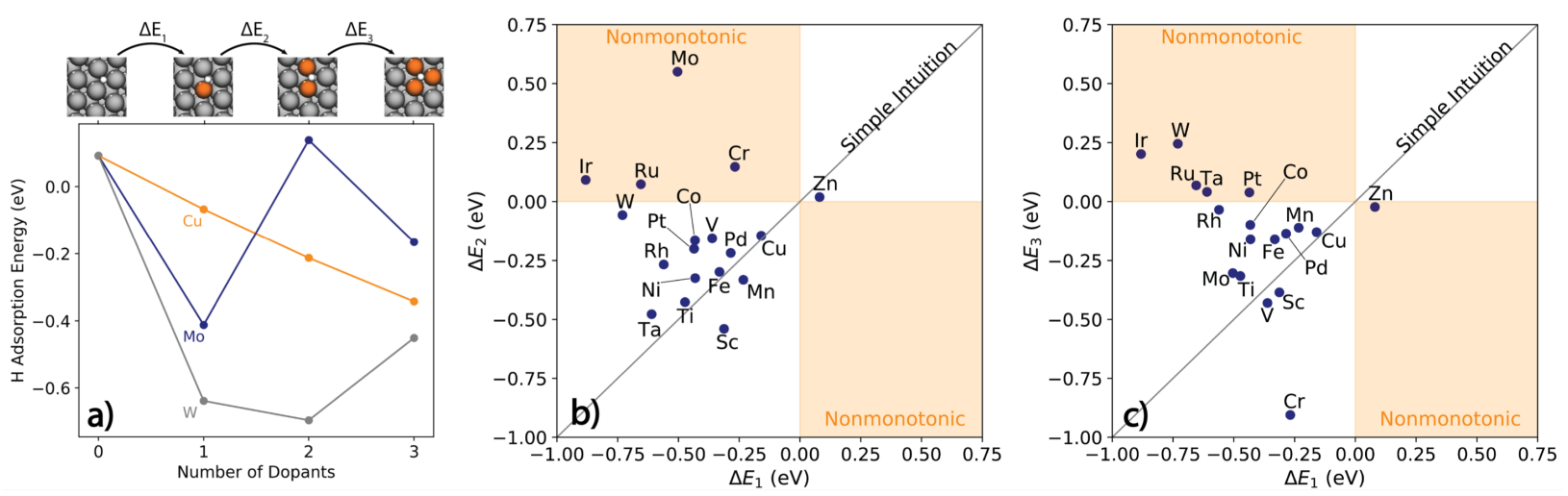

Figure 1 a) Hydrogen adsorption energy vs. number of dopants in a Ag surface. Graphics show relaxed geometries for $\mathrm{Cu}$ doped into $\mathrm{Ag}$. b) The difference in $\mathrm{H}$ adsorption energy between pure $\mathrm{Ag}$ and a single dopant $\left(\Delta \mathrm{E}_{1}\right)$ vs. the difference in adsorption energy between one dopant and two dopants $\left(\Delta \mathrm{E}_{2}\right)$. c) $\Delta \mathrm{E}_{1}$ vs. the difference in adsorption energy between two dopants and three dopants $\left(\Delta \mathrm{E}_{3}\right)$. In parts $\mathrm{b}$ and $\mathrm{c}$, the "Simple Intuition" line shows the case where adding a second or third dopant has the same effect on the adsorption energy as adding the first dopant.

More generally, we can elucidate trends by examining how the $\mathrm{H}$ adsorption energy differs between surfaces with consecutive numbers of dopants. We defined $\Delta \mathrm{E}_{1}, \Delta \mathrm{E}_{2}$, and $\Delta \mathrm{E}_{3}$ as the change in adsorption energy upon a change from zero to one, one to two, and two to three dopants, respectively (Figure 1a). Based on simple intuition, we generally expect that $\Delta \mathrm{E}_{1}, \Delta \mathrm{E}_{2}$, and $\Delta \mathrm{E}_{3}$ should be roughly similar. ${ }^{1-5}$ This is indeed the case for many of the dopant elements. However, we found that several dopants feature the unintuitive, nonmonotonic trends seen for Mo and $\mathrm{W}$ in Figure 1a. For Mo, $\mathrm{Ir}, \mathrm{Ru}$, and $\mathrm{Cr}, \Delta \mathrm{E}_{1}$ and $\Delta \mathrm{E}_{2}$ are quite different, and in fact have different signs (Figure 1b), corresponding to unexpected weakening of adsorption upon adding the second dopant atom. For Ir, W, Ru, Ta, and $\mathrm{Pt}, \Delta \mathrm{E}_{1}$ and $\Delta \mathrm{E}_{3}$ have different signs (Figure 1c), corresponding to unexpected weakening of adsorption upon adding the third dopant. Whether a particular dopant features a nonmonotonic trend appears to have no correlation to the absolute adsorption energy. For example, $\mathrm{Mo}_{1} \mathrm{Ag}$ and $\mathrm{Ti}_{1} \mathrm{Ag}$ have similar adsorption energies $(-0.41 \mathrm{eV}$ 
and $-0.38 \mathrm{eV}$, respectively), but $\mathrm{Mo}_{2} \mathrm{Ag}$ and $\mathrm{Ti}_{2} \mathrm{Ag}$ have adsorption energies that differ by nearly $1 \mathrm{eV}(0.14 \mathrm{eV}$ and $-0.81 \mathrm{eV})$.

These nonmonotonic trends were also observed for some dopants embedded in other inert metal surfaces (Figure 2). A given dopant generally behaves fairly similarly when embedded in $\mathrm{Cu}, \mathrm{Ag}$, or $\mathrm{Au}$, although there are some variations across hosts. For example, doping $\mathrm{Mo}$ and $\mathrm{Cr}$ into all three of these hosts gives nonmonotonic behavior, while doping Pd gives nearly linear behavior in all three cases. As a host, Pd behaves somewhat differently than $\mathrm{Au}, \mathrm{Ag}$, and $\mathrm{Cu}$, partially because it adsorbs $\mathrm{H}$ more strongly than the coinage metals. However, nonmonotonic behavior is still observed in some cases, such as Mo and Co. In these cases, adding a single dopant atom weakens $\mathrm{H}$ adsorption, but adding a second dopant atom strengthens it.
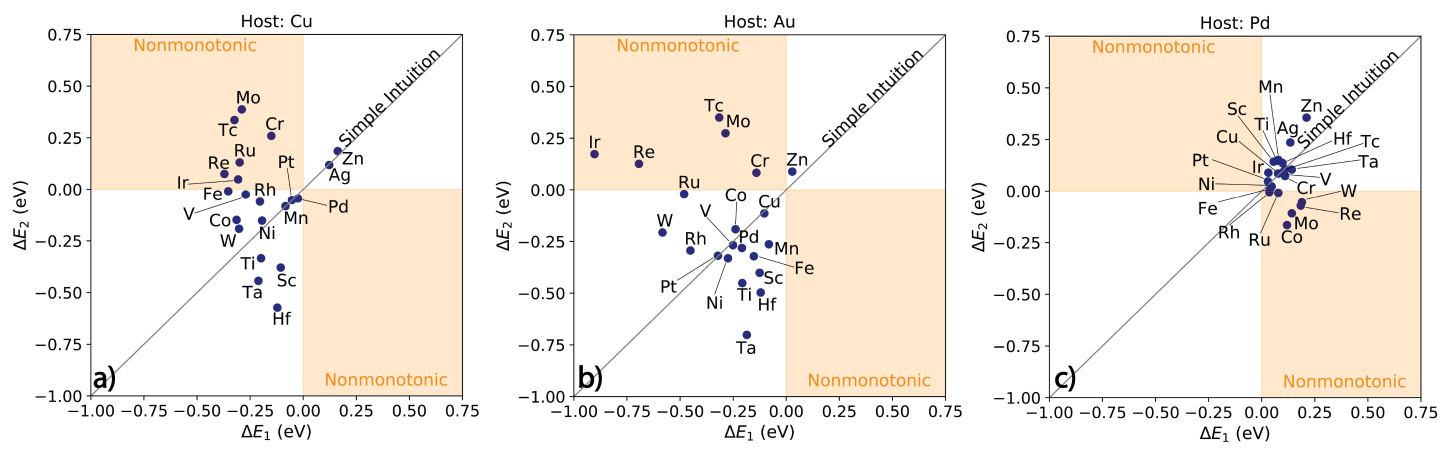

Figure 2. $\Delta \mathrm{E}_{2}$ vs. $\Delta \mathrm{E}_{1}$ for a) $\left.\mathrm{Cu}, \mathrm{b}\right) \mathrm{Au}$, and c) $\mathrm{Pd}$.

To understand the source of the nonmonotonic behavior, we examined the projected density of states (PDOS) on the dopant atoms. The dopant atom in single-atom alloys often features a single, sharp peak, somewhat similar to an electronic state in an atom in the gas-phase, as noted in previous work. ${ }^{12,17}$ We found that two-atom and three-atom ensembles also often have 
sharp peaks, but due to dopant-dopant hybridization and splitting there are often two peaks for dimers and three peaks for trimers (Figure 3a,b). There is an additional, broad feature that often appears in the PDOS between roughly $-6 \mathrm{eV}$ and $-3 \mathrm{eV}$ that is due to $\mathrm{d}$ states from neighboring Ag atoms, which appear in this energy range.
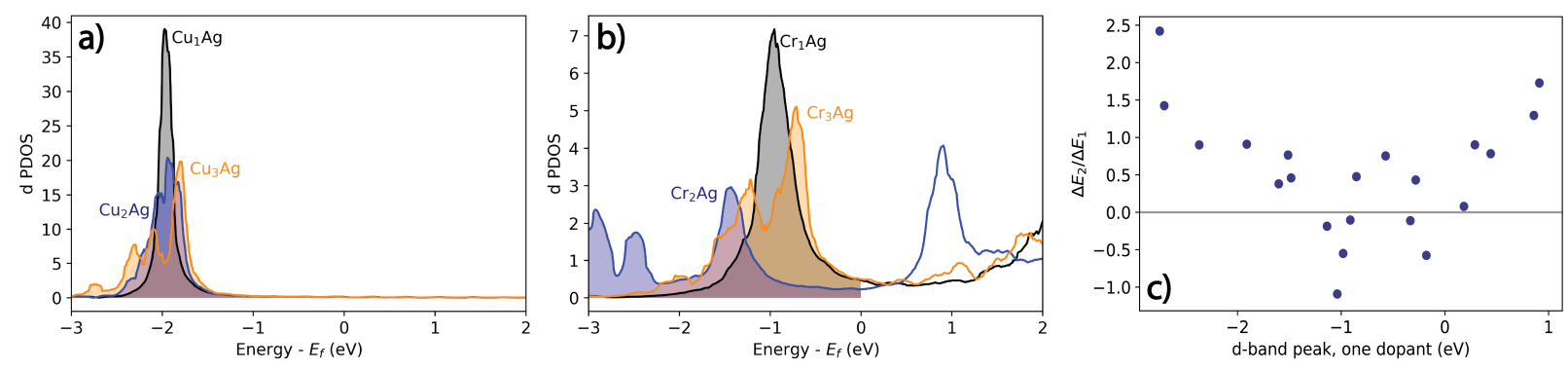

Figure 3. a) PDOS for the d-states on $\mathrm{Cu}$ atoms in $\mathrm{Cu}_{n} \mathrm{Ag}$; b) PDOS for the d-states on Mo atoms in $\mathrm{Mo}_{\mathrm{n}} \mathrm{Ag}$; c) $\Delta \mathrm{E}_{2} / \Delta \mathrm{E}_{1}$ vs. the d-band peak for the single-atom alloys, relative to the Fermi energy. Splitting of the d-band peaks strongly influences the changes in adsorption energy upon adding dopants.

Examination of the PDOS indicates that the splitting of these localized d states causes the nonmonotonic trends in adsorption energies. For the two-dopant cases, the d-state peaks can be split across the Fermi energy such that the upper state becomes unoccupied and the lower state has a low energy, as seen for $\mathrm{Mo}_{2} \mathrm{Ag}$ (Figure 3b). This results in a low d-band center and a low number of states near the Fermi energy, both of which are expected to lead to weak adsorption. ${ }^{21-}$ ${ }^{23}$ This splitting across the Fermi energy often occurs when the single-atom alloy's d-band peak is around $1 \mathrm{eV}$ below the Fermi Energy, such that nonmonotonic behavior is generally observed in these cases (Figure 3c). The precise amount of splitting and its precise effect on adsorption 
depends on the coupling and hybridization between dopant atoms and the hybridization between the dopant and the host metal atoms. This results in some variation in how $\Delta \mathrm{E}_{2} / \Delta \mathrm{E}_{1}$ depends on the d-band peak of the single-atom case, but nonmonotonic cases all have peaks within roughly 1 $\mathrm{eV}$ of the Fermi energy. We also found that $\Delta \mathrm{E}_{2} / \Delta \mathrm{E}_{1}$ can be linked to the difference in d-band center between the one-dopant and two-dopant cases, and that it has a similar trend as Figure 3c when plotted against the d-band center instead of the d-band peak (see Supporting Information). Hence, the behavior of the localized d-state peaks determines trends across the number of dopants, for a given dopant element.

While the d-band peaks and splitting explain trends across the number of dopant atoms, it is also important to understand trends across the type of dopant. For a given host, we found that the $\mathrm{H}$ adsorption energy is linked to the dopant's group in the periodic table (Figure 4). For transition metals, the dopant's group contains the same information as its idealized d-band filling, which has previously been found to be important in adsorption on transition metal alloys. ${ }^{21,24,25}$

The relationship between adsorption energy and dopant group is particularly distinct in single-atom alloys. Single dopants in the same periodic group often have similar adsorption energies, as shown by the darker, overlapping points in Figure 4a-d. For Ag-host and Cu-host surfaces, the exceptions to this similarity within a dopant group are nearly always cases where the surface has a magnetic moment. Most single-atom alloys that follow the primary, U-shaped relationship have a magnetic moment near 0 , while the outliers have magnetic moments up to approximately $4 \mu_{\mathrm{B}}$ (see Figure $\mathrm{S} 1$ ). For single-atom alloys with Au as the host, there is a systematic variation in adsorption energies within each group for groups 6 through 9. In this 
case, there is a smooth relationship for each period, with the $\mathrm{H}$ binding generally stronger as $5 \mathrm{~d}$ metals $>4 \mathrm{~d}$ metals $>3 \mathrm{~d}$ metals. This variation within each group may be due to Au's more extended d-orbitals, which may lead to higher hybridization with the dopant's orbitals and hence more variation among dopants of the same group.

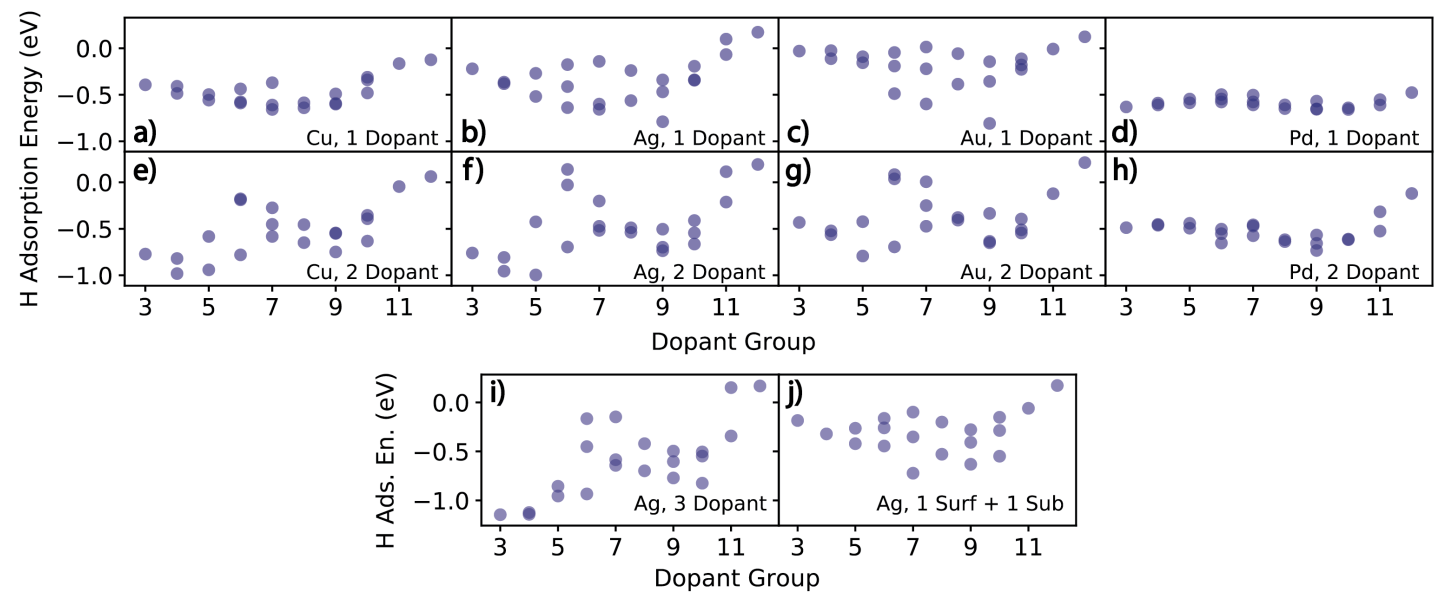

Figure 4. $\mathrm{H}$ adsorption energy as a function of dopant group in each host metal. a-d) 1 dopant surfaces; e-h) 2 dopant surfaces; i) 3 dopant surfaces; j) surfaces with a dopant in the surface and a neighboring dopant in the second layer.

When multiple dopant atoms are present, adsorption energies vary more within a group, making the relationship less smooth. Moreover, the relationship between the adsorption energy and dopant group is U-shaped for $\mathrm{Cu}$-host, Ag-host, and $\mathrm{Au}$-host single-atom alloy surfaces (Figure 4a-d). As the number of dopants increases, the adsorption energy vs. dopant group curve changes shape, such that dopants from the left side of the d-block adsorb $\mathrm{H}$ more strongly than those from the right side (Figure 4e-j). This is closer to the expected trend for pure metal surfaces. As the number of dopant atoms increases, the more the dopant peaks split and broaden, leading to more wider bands, more hybridization with the host, and more similar behavior to 
pure metals or bulk alloys. We also studied these trends for a Ag surface with one dopant in the surface layer, and one in the subsurface layer (Figure $4 \mathrm{j}$ ). In this case, there is still a U-shaped curve, but there is increased variation within a dopant group, likely due to dopant-dopant hybridization. For Pd-host surfaces, the adsorption energy curve is fairly flat and slightly Sshaped, with a similar shape for one and two dopants. Even in this case, there is more variation in adsorption energy within a group for the two-dopant surfaces than the single-atom alloy surfaces.

To understand how the electronic structure of the surface affects the adsorption strength, we calculated band properties based on the PDOS on the dopant atoms for the Ag-host bare surfaces with one, two, or three dopants. Broadly, these calculations suggest that the behavior of these systems is controlled by properties of the dopant's p-band and d-band, as suggested by previous work on transition metal alloy surfaces. ${ }^{21,24,26}$ However, the importance of these two bands changes for different numbers of dopants.

For single-atom alloys with a Ag host, variations in the adsorption energy are primarily controlled by the $\mathrm{p}$ band (Figure 5a,d). The p-band center correlates well with the adsorption energy $\left(R^{2}=0.73\right)$, while the d-band center correlates much less strongly with the adsorption energy $\left(R^{2}=0.37\right)$. Most of the correlation for the d-band center comes from the two points with the lowest d-band centers, $\mathrm{Au}_{1} \mathrm{Ag}$ and $\mathrm{Mn}_{1} \mathrm{Ag}$. Indeed, removing Au reduces the correlation for the d-band center on single-atom alloys to $R^{2}=0.16 . \mathrm{Zn}_{\mathrm{n}} \mathrm{Ag}$ and $\mathrm{Tc}_{\mathrm{n}} \mathrm{Ag}$ were removed from the d-band center plots because they are outliers, but including them does not change the qualitative trends (see Figure S5). As the number of dopants increases to two or three, the p-band center correlates less strongly with the adsorption energy, but the d-band center correlates more 
strongly. Further, the correlation between the adsorption energy and the p-band center is positive for single-atom alloys, but is negative for two or three dopants. Hence, adsorption on single-atom alloys has a qualitatively different dependence on the dopants' electronic structure than adsorption on larger ensembles. This change may be due to differences in the p-band DOS: for single-atom alloys, the $\mathrm{p}$ band generally has two broad peaks, while for larger ensembles the $\mathrm{p}$ band has many sharper peaks (Figure S7). The relationship between the p-band center and the adsorption energy for single-atom alloys also explains the $\mathrm{U}$ shape for the adsorption energy vs. group curve, as the p-band center has a U-shaped dependence on dopant group for single-atom alloys but decreases from left to right for two and three atom ensembles (Figure S6). Overall, the d-band model generally assumes a very broad, flat sp-band and that the d-band shape does not change too dramatically between surfaces ${ }^{23}$ and it is therefore reasonable that it may partially break down or behave differently for single-atom alloys.

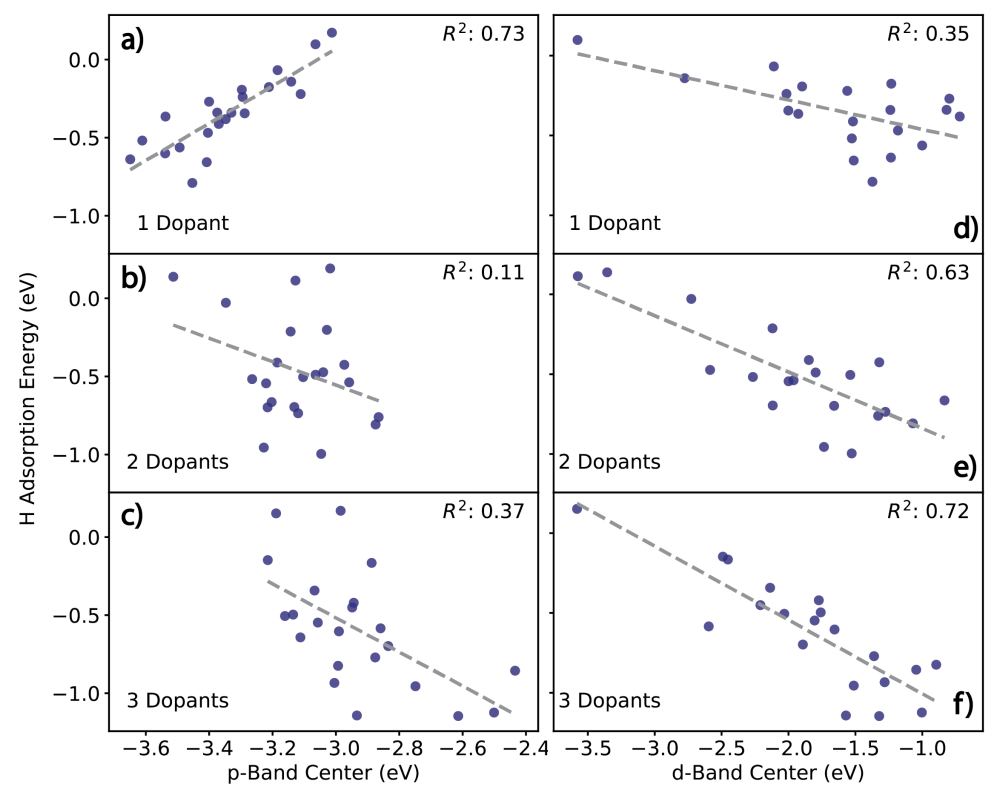

Figure 5. $\mathrm{H}$ adsorption energies on $\mathrm{Ag}$ surfaces doped with various metals as a function of a) $\mathrm{p}$ band center, and b) d-band center. Linear fits and corresponding $\mathrm{R}^{2}$ values are shown. The $\mathrm{p}$ - 
band controls adsorption for single-atom alloys, while the d-band controls adsorption for the three-dopant case.

Overall, the magnetic behavior of these embedded nanocluster systems can be somewhat complicated. In most cases that have a nonzero magnetic moment, adsorbed $\mathrm{H}$ somewhat reduces the magnetic moment relative to the bare surface (Figure S3), as seen in previous work on magnetic nanoclusters. ${ }^{27}$ In a few cases, such as $\mathrm{Mn}_{3} \mathrm{Ag}, \mathrm{H}$ has a very large effect on the magnetic moment. For Pd-host materials, the dopant appears to induce magnetism in surrounding Pd atoms in many cases. Further discussion is in the Supporting Information.

We also performed a few test calculations to compare $\mathrm{H}$ adsorption to $\mathrm{C}$ and $\mathrm{O}$ adsorption. These calculations suggest that trends for $\mathrm{C}$ and $\mathrm{O}$ across various dopants are similar, though not quite identical to, those for $\mathrm{H}$ (Figure S9). For example, Cu doped into Ag gives monotonic trends in adsorption energies for all three adsorbates, while Mo doped into Ag gives nonmonotonic trends for all three adsorbates.

In conclusion, we explain trends in adsorption on embedded nanoclusters, both across number of dopant atoms and across dopants. Counterintuitive nonmonotonic trends upon changing the number of dopants are due to splitting of localized electronic states. This is consistent with the well-known feature of supported or unsupported nanoclusters that properties can change significantly by adding a single atom. We find that single-atom alloys feature unusual behavior, even compared to larger embedded nanoclusters. Specifically, trends across the d-block are different, as the adsorption energy vs. dopant group curve is U-shaped for singleatom alloys instead of generally increasing from left to right as is the case for larger ensembles and pure metals. Single-atom alloys also have a different dependence on electronic structure: the p-band controls variations in adsorption energy more strongly than the d-band, while the d-band 
has a larger impact for larger ensembles and pure metals. Further, the relationship between the adsorption energy and the p-band center is positive for single-atom alloys but negative for two and three atom ensembles.

\section{Methods}

Plane-wave DFT calculations with the VASP $\operatorname{code}^{28,29}$ were used to obtain adsorption energies. The k-point mesh was $7 \times 7 \times 1$ with a $3 \times 3$ surface cell of 4 layers. The PBE exchange correlation functional was used, with the Tkatchenko-Scheffler method ${ }^{30}$ accounting for van der Waals interactions. The electronic convergence criterion was $10^{-5} \mathrm{eV}$. Adsorbates were placed in the fcc hollow site and allowed to fully relax to a force tolerance of 0.03 to $0.035 \mathrm{eV}$. Plane wave cut-offs were set to $400 \mathrm{eV}$, and all calculations were spin-polarized. To calculate the density of states, non-self-consistent calculations were performed with a fixed charge density and a 19x19x1 k-point grid. Atomic graphics were created using VMD. The p-band center was calculated only including states up to the Fermi energy, while the d-band center was calculated by including states up to $0.3 \mathrm{eV}$ above the Fermi energy, as in previous work..$^{21,24,26}$ The d-band peak was found by finding the energy with the maximum PDOS, after applying some mild smoothing. The center and peak energies are defined relative to the Fermi energy.

The ground-state magnetization states of these surfaces can be difficult to discover, and we often tested multiple initial magnetic states and various self-consistent field convergence algorithms. In some cases, we first performed a non-spin-polarized calculation and then used the charge density from this calculation as an input to the spin-polarized calculation to aid in convergence, or added penalty terms to bias the convergence towards a particular spin state. We then used the lowest energy spin state that we discovered in all cases. 


\section{ASSOCIATED CONTENT}

\section{Supporting Information.}

The following files are available free of charge.

Further discussion of magnetic effects, electronic structure effects and trends, and $\mathrm{C}$ and $\mathrm{O}$ adsorption energies. (PDF)

\section{AUTHOR INFORMATION}

\section{Notes}

The authors declare no competing financial interests.

\section{ACKNOWLEDGMENT}

We gratefully acknowledge support from Tulane University. A.M. acknowledges support from the National Science Foundation from grant DMR-1852274, and M.M.M. acknowledges support from the Louisiana Board of Regents Research Competitiveness Subprogram, Contract Number LEQSF(2019-22)-RD-A-20. This research was supported in part using computing resources provided by Technology Services at Tulane University, New Orleans, LA. Portions of this research were conducted with high performance computational resources provided by the Louisiana Optical Network Infrastructure (http://www.loni.org). We acknowledge useful discussions with E. C. H. Sykes.

\section{REFERENCES}

(1) Montemore, M. M.; Madix, R. J.; Kaxiras, E. How Does Nanoporous Gold Dissociate Molecular Oxygen? J. Phys. Chem. C 2016, 120, 16636-16640, DOI: 10.1021/acs.jpcc.6b03371. 
(2) Yu, W.-Y.; Zhang, L.; Mullen, G. M.; Henkelman, G.; Mullins, C. B. Oxygen Activation and Reaction on Pd-Au Bimetallic Surfaces. J. Phys. Chem. C 2015, 119, 11754-11762, DOI: $10.1021 /$ acs.jpcc.5b02970.

(3) Li, H.; Shin, K.; Henkelman, G. Effects of Ensembles, Ligand, and Strain on Adsorbate Binding to Alloy Surfaces. J. Chem. Phys. 2018, 149, DOI: 10.1063/1.5053894.

(4) Takehiro, N.; Liu, P.; Bergbreiter, A.; Nørskov, J. K.; Behm, R. J. Hydrogen Adsorption on Bimetallic PdAu(111) Surface Alloys: Minimum Adsorption Ensemble, Ligand and Ensemble Effects, and Ensemble Confinement. Phys. Chem. Chem. Phys. 2014, 16, 23930-23943, DOI: 10.1039/c4cp02589j.

(5) Choi, Y. M.; Compson, C.; Lin, M. C.; Liu, M. Ab Initio Analysis of Sulfur Tolerance of Ni, Cu, and Ni-Cu Alloys for Solid Oxide Fuel Cells. J. Alloys Compd. 2007, 427, 25-29, DOI: 10.1016/j.jallcom.2006.03.009.

(6) Montemore, M. M.; Cubuk, E. D.; Klobas, J. E. E.; Schmid, M.; Madix, R. J.; Friend, C. M.; Kaxiras, E. Controlling O Coverage and Stability by Alloying Au and Ag. Phys. Chem. Chem. Phys. 2016, 18, 26844-26853, DOI: 10.1039/C6CP05611C.

(7) Leon, C. C.; Lee, J. G.; Ceyer, S. T. Oxygen Adsorption on Au-Ni(111) Surface Alloys. J. Phys. Chem. C 2014, 118, 29043-29057, DOI: 10.1021/jp503758t.

(8) Liu, J.; Uhlman, M. B.; Montemore, M. M.; Trimpalis, A.; Giannakakis, G.; Shan, J.; Cao, S.; Hannagan, R. T.; Sykes, E. C. H.; Flytzani-Stephanopoulos, M. Integrated CatalysisSurface Science-Theory Approach to Understand Selectivity in the Hydrogenation of 1Hexyne to 1-Hexene on PdAu Single-Atom Alloy Catalysts. ACS Catal. 2019, 9, 8757- 
8765, DOI: $10.1021 /$ acscatal.9b00491.

(9) Kyriakou, G.; Boucher, M. B.; Jewell, A. D.; Lewis, E. A.; Lawton, T. J.; Baber, A. E.; Tierney, H. L.; Flytzani-Stephanopoulos, M.; Sykes, E. C. H. Isolated Metal Atom Geometries as a Strategy for Selective Heterogeneous Hydrogenations. Science 2012, 335, 1209-1212, DOI: 10.1126/science.1215864.

(10) Giannakakis, G.; Flytzani-Stephanopoulos, M.; Sykes, E. C. H. Single-Atom Alloys as a Reductionist Approach to the Rational Design of Heterogeneous Catalysts. Acc. Chem. Res. 2019, 52, 237-247, DOI: 10.1021/acs.accounts.8b00490.

(11) Saxena, S.; Khan, T. S.; Jalid, F.; Ramteke, M.; Haider, M. A. In Silico High Throughput Screening of Bimetallic and Single Atom Alloys Using Machine Learning and Ab Initio Microkinetic Modelling . J. Mater. Chem. A 2019, DOI: 10.1039/c9ta07651d.

(12) Greiner, M. T.; Jones, T. E.; Beeg, S.; Zwiener, L.; Scherzer, M.; Girgsdies, F.; Piccinin, S.; Armbrüster, M.; Knop-Gericke, A.; Schlögl, R. Free-Atom-like d States in SingleAtom Alloy Catalysts. Nat. Chem. 2018, 10, 1008-1015, DOI: 10.1038/s41557-018-01255.

(13) Dasgupta, A.; Gao, Y.; Broderick, S. R.; Pitman, E. B.; Rajan, K. Machine Learning Aided Identification of Single Atom Alloy Catalysts. J. Phys. Chem. C 2020, DOI: 10.1021/acs.jpcc.0c01492.

(14) Darby, M. T.; Stamatakis, M.; Michaelides, A.; Sykes, E. C. H. Lonely Atoms with Special Gifts: Breaking Linear Scaling Relationships in Heterogeneous Catalysis with Single-Atom Alloys. J. Phys. Chem. Lett. 2018, 9, 5636-5646, DOI: 
10.1021/acs.jpclett.8b01888.

(15) Montemore, M. M.; Medlin, J. W. Site-Specific Scaling Relations for Hydrocarbon Adsorption on Hexagonal Transition Metal Surfaces. J. Phys. Chem. C 2013, 117, 2007820088, DOI: $10.1021 / j p 4076405$.

(16) Sun, G.; Zhao, Z.J.; Mu, R.; Zha, S.; Li, L.; Chen, S.; Zang, K.; Luo, J.; Li, Z.; Purdy, S. C.; Kropf, A. J.; Miller, J. T.; Zeng, L.; Gong, J. Breaking the Scaling Relationship via Thermally Stable $\mathrm{Pt} / \mathrm{Cu}$ Single Atom Alloys for Catalytic Dehydrogenation. Nat. Commun. 2018, 9, 4454, DOI: 10.1038/s41467-018-06967-8.

(17) Thirumalai, H.; Kitchin, J. R. Investigating the Reactivity of Single Atom Alloys Using Density Functional Theory. Top. Catal. 2018, 0, 1-13, DOI: 10.1007/s11244-018-0899-0.

(18) Fung, V.; Hu, G.; Sumpter, B. Electronic Band Contraction Induced Low Temperature Methane Activation on Metal Alloys. J. Mater. Chem. A 2020, 8, 6057-6066, DOI: 10.1039/D0TA00375A.

(19) Jin, R.; Zeng, C.; Zhou, M.; Chen, Y. Atomically Precise Colloidal Metal Nanoclusters and Nanoparticles: Fundamentals and Opportunities. Chem. Rev. 2016, 116, 1034610413, DOI: 10.1021/acs.chemrev.5b00703.

(20) Hoyt, R. A.; Montemore, M. M.; Fampiou, I.; Chen, W.; Tritsaris, G.; Kaxiras, E. Machine Learning Prediction of $\mathrm{H}$ Adsorption Energies on Ag Alloys. J. Chem. Inf. Model. 2019, 59, 1357-1365, DOI: 10.1021/acs.jcim.8b00657.

(21) Montemore, M. M.; Medlin, J. W. A Unified Picture of Adsorption on Transition Metals 
through Different Atoms. J. Am. Chem. Soc. 2014, 136, 9272-9275, DOI: $10.1021 / \mathrm{ja} 504193 \mathrm{w}$.

(22) Rangan, M.; Yung, M. M.; Medlin, J. W. NiW and NiRu Bimetallic Catalysts for Ethylene Steam Reforming: Alternative Mechanisms for Sulfur Resistance. Catal. Letters 2012, $142,718-727$.

(23) Hammer, B.; Nørskov, J. K. Theoretical Surface Science and Catalysis -Calculations and Concepts. In Advances in Catalysis; Elsevier, 2000; Vol. 45, pp 71-129, DOI: 10.1016/S0360-0564(02)45013-4.

(24) Montemore, M. M.; Medlin, J. W. Predicting and Comparing C--M and O--M Bond Strengths for Adsorption on Transition Metal Surfaces. J. Phys. Chem. C 2014, 118, 2666-2672, DOI: 10.1021/jp5001418.

(25) Montemore, M.; Nwaokorie, C. F.; Kayode, G. General Screening of Surface Alloys for Catalysis. Catal. Sci. Technol. 2020, DOI: 10.1039/D0CY00682C.

(26) Montemore, M. M.; Nwaokorie, C. F.; Kayode, G. O. General Screening of Surface Alloys for Catalysis. chemRxiv 2020, DOI: 10.26434/chemrxiv.11704920.v2, DOI: 10.26434/chemrxiv.11704920.v2.

(27) Hoyt, R. A.; Montemore, M. M.; Kaxiras, E. Nonadiabatic Hydrogen Dissociation on Copper Nanoclusters. J. Phys. Chem. Lett. 2018, 9, 5339-5343, DOI: 10.1021/acs.jpclett.8b02133.

(28) Kresse, G.; Furthmüller, J. Efficiency of Ab-Initio Total Energy Calculations for Metals 
and Semiconductors Using a Plane-Wave Basis Set. Comput. Mater. Sci. 1996, 6, 15-50, DOI: 10.1016/0927-0256(96)00008-0.

(29) Kresse, G.; Hafner, J. Ab Initio Molecular Dynamics for Liquid Metals. Phys. Rev. B 1993, 47, 558-561, DOI: 10.1103/PhysRevB.47.558.

(30) Tkatchenko, A.; Scheffler, M. Accurate Molecular Van Der Waals Interactions from Ground-State Electron Density and Free-Atom Reference Data. Phys. Rev. Lett. 2009, 102, 073005, DOI: 10.1103/PhysRevLett.102.073005. 